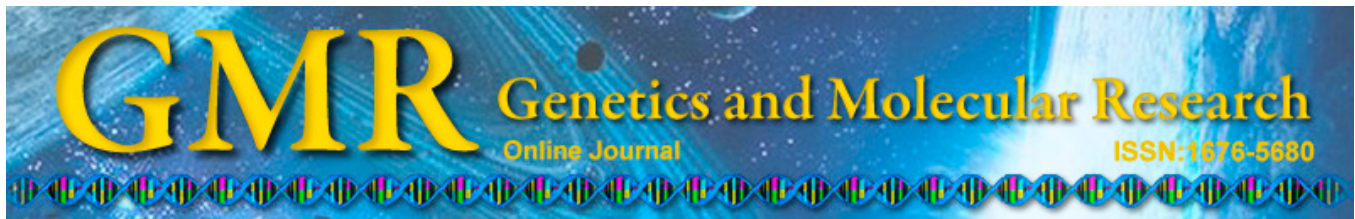

\title{
Correlation of spicule sign on computed tomography scans with peripheral lung cancers associated with interstitial lung disease and chronic obstructive pulmonary disease
}

\author{
L. Chen, L. Gao and W.L. Wu \\ Department of Radiology, Longgang Central Hospital of Shenzhen, \\ Shenzhen, Guangdong Province, China \\ Corresponding author: L. Chen \\ E-mail: c16541@163.com
}

Genet. Mol. Res. 14 (1): 2234-2240 (2015)

Received January 30, 2014

Accepted July 2, 2014

Published March 27, 2015

DOI http://dx.doi.org/10.4238/2015.March.27.9

\begin{abstract}
The objective of this study was to investigate the correlation between spicular signs on computed tomography (CT) scans and peripheral lung cancer (PLC) that is associated with interstitial lung disease (ILD) and chronic obstructive pulmonary disease (COPD). We analyzed clinical data from 96 PLC cases and grouped patients based on whether they had interstitial pneumonia into either ILD/COPD group or non-ILD/COPD group. The occurrence rate of spicule sign was $90.3 \%$ in the ILD/COPD group and $61.8 \%$ in the non-ILD/COPD group, respectively. There was a significant difference between these groups $(\mathrm{P}<0.05)$. There were no significant differences in the occurrence rate of spicular signs among patients with different pathological types of PLC. The severity of ILD affected the spicular morphology on CT scans directly. There was a significant correlation between the appearance of spicule sign on CT scans and PLC that was associated with ILD/COPD.
\end{abstract}

Key words: Chronic obstructive pulmonary disease; Spicule sign; Computed tomography; Interstitial lung disease; Peripheral lung cancer 
Spiculation sign on lung cancer associated with ILD and COPD

\section{INTRODUCTION}

Peripheral lung cancer (PLC) is a type of carcinoma that usually occurs at the inferior segment of the bronchia. In radiographic imaging, PLC usually appears as a nodule that is easily confused with surrounding inflammatory nodules and tubercle (Stiewe, 2007; De Sousa et al., 2012). Following the development of imaging technology, current computed tomography (CT) techniques can obtain 3 dimensional (3D) images, which is different from the previous methods as cross-section scanning. Current techniques also allow us to observe the PLC diagnostic parameters, such as spicular signs, lobulation and spiculate protuberance. Spicule sign on CT image is an important index for PLC diagnosis (Swisher et al., 1999; Mitsudomi et al., 2000; Rho et al., 2007). Related studies have indicated that interstitial lung disease (ILD) and chronic obstructive pulmonary disease (COPD) significantly correlate with spicule sign on CT images of PLC (Kennedy et al., 1997). In the present study, we sought to clarify this correlation by analyzing clinical data from 96 PLC patients in our hospital.

\section{MATERIAL AND METHODS}

\section{Clinical data}

We retrospectively analyzed clinical data from 96 patients diagnosed with PLC in our hospital between May 2012 and June 2013. The patients were divided into ILD/COPD group $(\mathrm{N}=62)$ and non-ILD/COPD group $(\mathrm{N}=34)$ based on whether they had interstitial pneumonia or not. The ILD/COPD group included 41 males and 21 females with ages ranging from 41 to 79 years (means $\pm \mathrm{SD}, 56.2 \pm 10.3$ years). Tumor sizes ranged from 1.3 to $4.7 \mathrm{~cm}$ (means \pm $\mathrm{SD}, 2.6 \pm 1.3 \mathrm{~cm}$ ). The pathology types of these tumors included bronchoalveolar carcinoma (9 patients), small cell carcinoma (10 patients), squamous cell carcinoma (14 patients), and adenocarcinoma (29 patients). The non-ILD/COPD group included 18 males and 16 females with ages ranging from 42 to 78 years (means \pm SD, $55.6 \pm 10.7$ years). The tumor sizes in this group ranged from 1.2 to $4.5 \mathrm{~cm}$ (means $\pm \mathrm{SD}, 2.3 \pm 1.1 \mathrm{~cm}$ ). The pathology types of the tumors in this group included bronchioloalveolar carcinoma (5 patients), small cell carcinoma (7 patients), squamous cell carcinoma (10 patients), and adenocarcinoma (12 patients).

Diagnostic criteria were based on Pal et al. (2001), Katzenberger et al. (2006), and Murakami et al. (2010). Diagnoses were made by experienced doctors after examination of CT images. ILD was indicated by the presence of abnormal pulmonary mesenchyma, nodules, hypertrophy, and fibrosis. COPD was characterized by partial or complete pulmonary emphysema with clinical symptoms including shortness of breath, chest tightness, fever, and hemoptysis (Joshi et al., 2005; Gentile et al., 2006; Battaglia et al., 2006; Wohlfert and Belkaid, 2010).

Patients were excluded from the study if there was incomplete clinical data, unmatched research regulations, severe adverse reaction, or died during treatment.

\section{Measurements}

Regular scans were performed using 64-slice spiral CT. Ultravist solution (360 mg/ $\mathrm{mL}$ ) was used as a non-ionic contrast (Lee and Mazmanian, 2010). The scanning parameters were: $0.723 \mathrm{~mm}$ slice thickness, $0.723 \mathrm{~mm}$ slice interval, $120 \mathrm{kV}$ voltage, and 276 to $700 \mathrm{~mA}$ current (Weaver and Hatton, 2009). Multiplanar reconstruction, maximum intensity projection 
and curved three-dimensional reconstruction was used for 3-D mapping. Four-chambers, long axis, and short axis were fixed during scanning (Coombes et al., 2007).

\section{Observation of spicule sign on CT images}

The feature of spicule sign on CT images of PLC were diffuse and demonstrated independent linearity. They originated from the margin of the carcinoma, extended into the pulmonary parenchyma, and separated from the thoracic diaphragm (Murai et al., 2010; Hadis et al., 2011; Miyao et al., 2012). Spicule sign that were clear and countable were recorded as numbers, while spicule sign with serious divergencyor cactus-like diffusion were quantified as area. Observed data was converted based on the width and length with the boundary as 2 $\mathrm{mm}$ and $5 \mathrm{~mm}$, respectively (Busch et al., 1998). Evaluation of spicule sign was performed by 2 doctors with significant clinical experience in a double-blinded manner. Any inconsistencies were discussed before a final decision was made (Lahl et al., 2007).

\section{Statistical analysis}

All data are reported as means \pm standard deviation. Results were analyzed using SPSS, version 19.0 (IBM Corp., Armonk, NY, USA). Categorical data were analyzed with the chi-square test while measurement data were analyzed by the Student $t$-test. Results with $\mathrm{P}$ $<0.05$ were considered to be statistically significant.

\section{RESULTS}

\section{Comparison of presence of spicule sign between 2 groups}

The ILD/COPD group included 56 patients with spicule sign (90.3\%) and 6 patients with no spicule sign $(9.7 \%)$. The non-ILD/COPD group included 21 patients with spicule sign $(61.8 \%)$ and 13 patients with no spicule sign (38.2\%). Occurrence rate of spicule sign was significantly higher in the ILD/COPD group compared to the non-ILD/COPD group $(\mathrm{P}<0.05)$, suggesting a correlation between spicule sign and ILD/COPD (Table 1).

Table 1. Comparison of spicule sign between groups.
\begin{tabular}{lcccc}
\hline Spicule sign & \multicolumn{2}{c}{ ILD/COPD group (N=62) } & & \multicolumn{2}{c}{ Non-ILD/COPD group (N = 34) } \\
\cline { 2 - 5 } & Cases & Rate (\%) & Cases & Rate (\%) \\
\hline+ & 56 & 90.3 & 21 & 61.8 \\
- & 6 & 9.7 & 13 & 38.2 \\
\hline
\end{tabular}

$\mathrm{COPD}=$ chronic obstructive pulmonary disease; ILD = interstitial lung disease.

\section{Correlation between spicule sign rate and PLC types in the ILD/COPD group}

In the ILD/COPD group, spicule sign occurred in $93.1 \%$ of the adenocarcinomas, $85.7 \%$ of the squamous cell carcinomas, $77.8 \%$ of the bronchioloalveolar carcinomas, and $10 \%$ of the small cell carcinomas. Chi-square tests showed that there were no significant differences among the different pathology types $\left(\chi^{2}=7.56, \mathrm{P}>0.05\right.$; Table 2$)$. Typical CT imagesare shown in Figures 1-4. 
Table 2. Comparison of spicule sign occurrence in different pathological types of PLC in the ILD/COPD group.

\begin{tabular}{lrcc}
\hline Pathological type & N & Spicule sign $(+)$ cases & Rate $(\%)$ \\
\hline Adenocarcinoma & 29 & 27 & 93.1 \\
Squamous cell carcinoma & 14 & 12 & 85.7 \\
Bronchioloalveolar carcinoma & 9 & 7 & 77.8 \\
Small cell carcinoma & 10 & 1 & 10 \\
\hline
\end{tabular}

$\mathrm{COPD}=$ chronic obstructive pulmonary disease; ILD = interstitial lung disease; PLC = peripheral lung cancer.

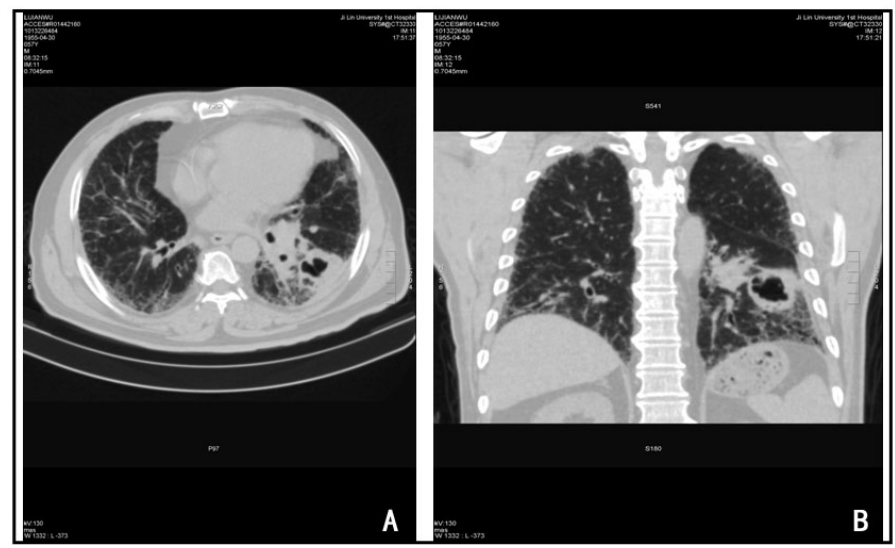

Figure 1. Images from a 57-year-old male, with a history of cough, expectoration, and dyspnea for 2 months. The sign aggravated during activity and prostration. Computed tomography revealed irregular cavitary lesions in the inferior lobe of the left lung with uneven thickness of the wall and wall nodule. Sparse speculation and superficial lobulation surround the tumor with adjacent pleura adhesion and thicken. Increased intensity of the parenchyma in whole lungs, thickened interlobular septum, and ground-glass-like high intensity opacity was observed. Sputum cytology revealed that the patient had squamous cell carcinoma.

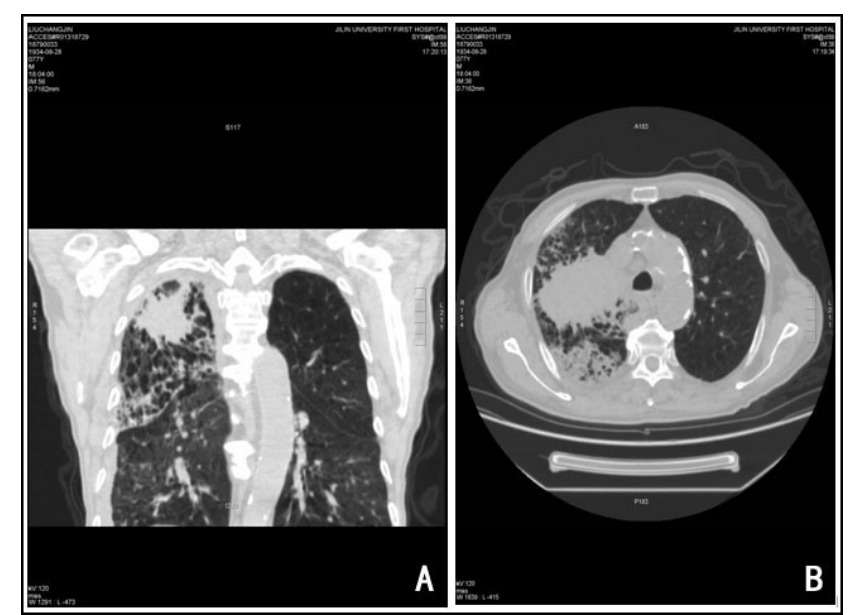

Figure 2. Images from a 77-year-old male with a history of intermittent cough, expectoration and wheeze for 30 years, and aggravate for 1 month. Computed tomography (CT) revealed a crumb soft tissue shadow in the superior lobe of the right lung, surrounded by large spiculation. Thickened interlobular septum appeared in the right lung, while honey combing appeared in the pulmonary parenchyma. Pathological examination found adenocarcinoma invasion in a biopsy specimen. Positron emission tomography-CT identified a high-metabolic mass in the superior lobe of the right lung, which was suggestive of PLC. 


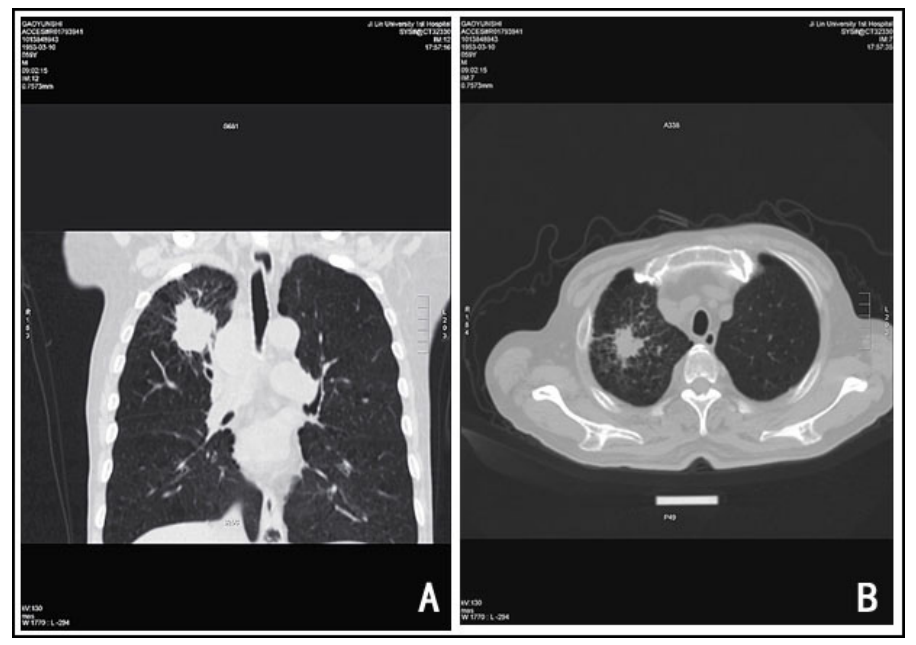

Figure 3. Images from a 59-year-old male with a history of a mass in the superior lobe of the right lung for 9 months with progressive enlargement. Computed tomography revealed deep lobulation, spiculation and spiculate protuberance surround the mass in the superior lobe of the right lung, enhanced transmittance in the pulmonary parenchyma, and multiple small flaky lucent areas with low intensity but no pulmonary stripe. Pathology identified the lesion as adenocarcinoma of the superior lobe of the right lung.

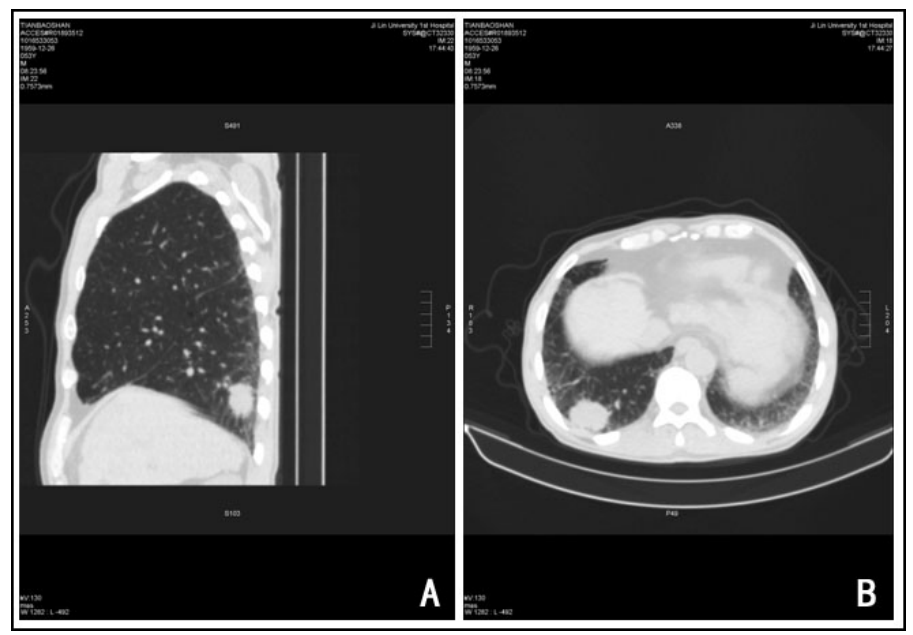

Figure 4. Images from a 53-year-old male with a history of a space-occupying lesion in the inferior lobe of the right lung. Pathology examination identified the lesion as non-small cell lung cancer, trending to squamous cell carcinoma. Computed tomography revealed a crumby shadow of soft tissue in the inferior lobe of the right lung with superficial lobulated edge and small speculation.

\section{DISCUSSION}

ILD initially arises from inflammation of alveoli, gradually affects alveolar blood vessels and the surrounding tracheal bronchus, and results in self-repair by the pulmonary mesenchyma through fibrosis (Billerbeck et al., 2007). COPD is an irreversible airflow disease 
that results in pulmonary emphysema, enhancement and thickening of the pulmonary interstitium, and fibrosis due to the repair process. Related studies have indicated that fibrosis of the pulmonary interstitium is a sign of pulmonary lesion repair. CT images of IPD and COPD reveal the presence of pulmonary interstitial fibrosis and hypertrophy as well as pulmonary emphysema. Many studies have found that pulmonary interstitial fibrosis, hypertrophy and pulmonary emphysema are accompanied by spicule sign on CT images, while spicule sign is also a key index for CT measurement of PLC (Suvas et al., 2003). Therefore, it is logical to hypothesize that ILD and COPD correlate with spicule sign on CT images of PLC. In the present study, we retrospectively analyzed data from 96 PLC patients treated in our hospital between May 2012 and June 2013 and found that IPD and COPD significantly correlated with spicule sign on CT images of PLC.

The present study found the positive rate for spicule sign was $90.3 \%$ in the ILD/ COPD group and $61.8 \%$ in the non-ILD/COPD group. There was a significant difference between these groups, suggesting a correlation between spicule sign on CT images of PLC and ILD/COPD. Chi-square tests indicated that there were no significant differences among different pathological types of PLC with regard to the positive rate of spicule sign on CT images, suggesting that there were no correlations between spicule sign and PLC pathological types.

In summary, spicule sign on CT images of PLC were significantly correlated with ILD and COPD, providing a reliable basis for the diagnosis of PLC in the clinic that should be more widely used.

\section{Conflicts of interest}

The authors declare no conflict of interest.

\section{REFERENCES}

Battaglia M, Gregori S, Bacchetta R and Roncarolo MG (2006). Tr1 cells: from discovery to their clinical application. Semin. Immunol. 18: 120-127.

Billerbeck E, Böttler T and Thimme R (2007). Regulatory T cells in viral hepatitis. World J. Gastroenterol. 13: 4858-4864.

Busch DH, Pilip IM, Vijh S and Pamer EG (1998). Coordinate regulation of complex T cell populations responding to bacterial infection. Immunity 8: 353-362.

Coombes JL, Siddiqui KRR, Arancibia-Cárcamo CV, Hall J, et al. (2007). A functionally specialized population of mucosal CD103+ DCs induces Foxp3+ regulatory T cells via a TGF- $\beta$ - and retinoic acid-dependent mechanism. $J$. Exp. Med. 204: 1757-1764.

De Sousa WAT, Rodrigues LV, da Silva Jr RG and Vieira FL (2012). Immunohistochemical evaluation of p53 and ki-67 proteins in colorectal adenomas. Arq. Gastroenterol. 49: 35-40.

Gentile V, Vicini P, Giacomelli L, Cardillo MR, et al. (2006). Detection of human papillomavirus DNA, p53 and ki67 expression in penile carcinomas. Int. J. Immunopathol. Pharmacol. 19: 209-215.

Hadis U, Wahl B, Schulz O, Hardtke-Wolenski M, et al. (2011). Intestinal tolerance requires gut homing and expansion of Foxp $3^{+}$regulatory $\mathrm{T}$ cells in the lamina propria. Immunity 34: 237-246.

Joshi A, Garg G, Tompkins MB and Tompkins WA (2005). Preferential feline immunodeficiency virus (FIV) infection of CD4+ CD25+ T-regulatory cells correlates both with surface expression of CXCR4 and activation of FIV long terminal repeat binding cellular transcriptional factors. J. Virol. 79: 4965-4976.

Katzenberger T, Petzoldt C, Höller S, Mäder U, et al. (2006). The ki67 proliferation index is a quantitative indicator of clinical risk in mantle cell lymphoma. Blood 107: 3407-3407.

Kennedy MM, Lamb D, King G and Kerr KM (1997). Cell proliferation, cell loss and expression of bcl-2 and p53 in human pulmonary neoplasms. Br. J. Cancer 75: 545-547.

Lahl K, Loddenkemper C, Drouin C, Freyer J, et al. (2007). Selective depletion of Foxp3 ${ }^{+}$regulatory T cells induces a scurfy-like disease. J. Exp. Med. 204: 57-63. 
Lee YK and Mazmanian SK (2010). Has the microbiota played a critical role in the evolution of the adaptive immune system? Science 330: 1768-1773.

Mitsudomi T, Hamajima N, Ogawa M and Takahashi T (2000). Prognostic significance of p53 alterations in patients with non-small cell lung cancer: a meta-analysis. Clin. Cancer Res. 6: 4055-4063.

Miyao T, Floess S, Setoguchi R, Luche H, et al. (2012). Plasticity of Foxp3 ${ }^{+}$T cells reflects promiscuous Foxp3 expression in conventional T cells but not reprogramming of regulatory T cells. Immunity 36: 262-275.

Murai M, Krause P, Cheroutre H and Kronenberg M (2010). Regulatory T-cell stability and plasticity in mucosal and systemic immune systems. Mucosal Immunol. 3: 443-449.

Murakami S, Saito H, Sakuma Y, Mizutani Y, et al. (2010). Correlation of ${ }^{18} \mathrm{~F}$-fluorodeoxyglucose uptake on positron emission tomography with ki-67 index and pathological invasive area in lung adenocarcinomas $30 \mathrm{~mm}$ or less in size. Eur. J. Radiol. 75: e62-e66.

Pal S, Datta K and Mukhopadhyay D (2001). Central role of p53 on regulation of vascular permeability factor/vascular endothelial growth factor (VPF/VEGF) expression in mammary carcinoma. Cancer Res. 61:6952-6957.

Rho JK, Choi YJ, Ryoo BY, Na II, et al. (2007). p53 enhances gefitinib-induced growth inhibition and apoptosis by regulation of Fas in non-small cell lung cancer. Cancer Res. 67: 1163-1169.

Stiewe T (2007). The p53 family in differentiation and tumorigenesis. Nat. Rev. Cancer 7: 165-167.

Suvas S, Kumaraguru U, Pack CD, Lee S, et al. (2003). $C D 4^{+} \mathrm{CD} 25^{+} \mathrm{T}$ cells regulate virus-specific primary and memory CD8 ${ }^{+}$T cell responses. J. Exp. Med. 198:889-901.

Swisher SG, Roth JA, Nemunaitis J, Lawrence DD, et al. (1999). Adenovirus-mediated p53 gene transfer in advanced non-small-cell lung cancer. J. Natl. Cancer Inst. 91: 763-771.

Weaver CT and Hatton RD (2009). Interplay between the $\mathrm{T}_{\mathrm{H}} 17$ and $\mathrm{T}_{\text {reg }}$ cell lineages: a (co-)evolutionary perspective. Nat. Rev. Immuol. 9: 883-889.

Wohlfert E and Belkaid Y (2010). Plasticity of $\mathrm{T}_{\text {reg }}$ at infected sites. Mucosal Immunol. 3: 213-215. 